\title{
What's in a name? That which we call sacroiliitis by any other name would look the same...
}
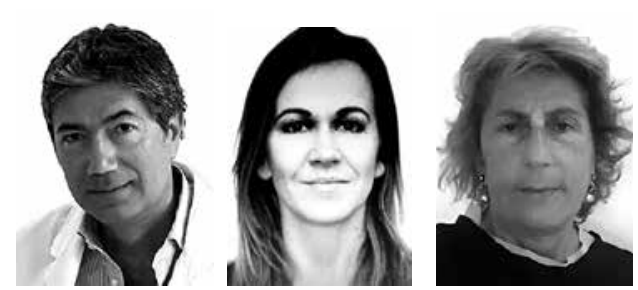

\author{
Alfredo Tarantino, Justyna Paulina Jablonska, Paola D'Aprile \\ Department of Radiology, San Paolo Hospital, Bari, Italy
}

"What's in a name? That which we call a rose By any other name would smell as sweet." Romeo and Juliet (II, 2, 1-2)

Since the appearance of magnetic resonance imaging (MRI), the diagnostic rates of sacroiliac joint (SIJ) changes have increased rapidly.

The term sacroiliitis is generally used to indicate oedema of the SIJ of rheumatic origin, but similar oedematous lesions may be of non-rheumatic origin, even more frequently, in patients with chronic low back pain. Distinguishing rheumatic from non-rheumatic sacroiliitis is very important in terms of therapeutic choice. In particular, overdiagnosis of rheumatic sacroiliitis may result in the prescription of expensive and inappropriate treatments. Therefore, the term "sacroiliitis" may possibly be unsuitable in the near future.

Let us start by asking "what is inflammation?" Inflammation is, generally speaking, the body's immune system's response to a stimulus. Inflammation happens when the immune system fights against something that may turn out to be harmful. Inflammatory conditions always have a name ending with "-itis", as in this case: sacroiliitis. We can divide them into rheumatic and non-rheumatic, such as mechanical and infective.

Diagnostics of rheumatic sacroiliitis, which is the primary feature and a hallmark of axial spondyloarthritis, is primarily based on clinical examination and laboratory testing. The clinical criterion used by rheumatologists when referring patients for accessory examinations (laboratory or radiological), is chronic back pain [1].

According to the Assessment of Spondyloarthritis International Society (ASAS) criteria, four types of inflammatory lesions in sacroiliitis associated with spondyloarthritis (SpA) can be identified: bone marrow oedema and osteitis, synovitis, enthesitis and capsulitis.
Bone oedema and osteitis are indispensible to diagnose active sacroiliitis only [2].

Bone marrow oedema is detected as areas of increased signal intensity in 12 -weighted images with fat saturation or short $\tau$ inversion recovery sequences (STIR), and is interpreted as an acute inflammatory lesion. It is usually hypointense in T1-weighted sequences. In T1-weighted sequences with fat suppression after the i.v. injection of paramagnetic contrast it shows an enhacement due to inereased of vascularization and reactive perfusion to inflammation and it is categorized as osteitis [3].

To diagnose sacroiliitis it was established by consensus that one area of bone oedema/osteitis should be present in at least two consecutive cuts, but if there is more than one focus, one cut would be enough regardless of its size in both cases. ASAS criteria do not establish requirements or stipulations as regards the distribution of lesions [4].

Studies of the subject in the literature have demonstrated that mechanical sacroiliitis is a markedly unrecognized condition and, interestingly, is 20 to 100 times more common than rheumatic sacroiliitis [5].

The SIJs are essential for effectively transferring loads between the spine and legs. The sacrum, pelvis and spine, and the connections to the arms, legs and head are functionally related through muscular, fascial and ligamentous interconnections. Likewise, efficient motor control does not provide a solution for individual joints, but orchestrates efficient reaction forces to integrate and stabilize the kinetics of our body [6]. Therefore transmission of intra- and extramuscular myofascial 
forces in the locomotor system is essential for the correct clinical diagnosis.

Radiologically speaing, MR signal of BMO areas is the in rheumatic and nonreumatic sacroilitis, even if, as we recently observed, it ofiffers in extent and locafion.

Septic sacroiliitis is extremely rare. Diagnosis of a sacroiliac joint infection is facilitated by specific physical findings, including fever, and laboratory values [7].

At this point the correctness of the term sacroiliitis must be discussed. In the strict sense of the word, by sacroiliitis we conventionally mean an inflammatory process involving one or both sacroiliac joints. Therefore, some difficulty has already arisen in calling non-rheumatic changes "sacroiliitis". Many oedematous processes involving the sacroiliac joints emerge in the literature as well in our everyday practice, so it seems to be required to clarify this nomenclature estimating the pathogenesis of this phenomenon. Inflammation is the reaction of the vascular and supporting elements to injury, and it results in the formation of a macrophageand lymphocyte-rich exudate. The number of studies on histology of sacroiliac inflammation has remained rather limited for decades, because access to the SIJ is difficult. There is one report in the English language literature of Bollow et al. They introduced the technique of computed tomography-guided sacroiliac biopsy performed in combination with anti-inflammatory intraarticular treatment for immuno-histological analysis of patients with sacroiliitis. Their study showed that T cells and macrophages are the predominant, but aspecific, cells in patients with active sacroiliitis. To date, no studies have been performed to determine which cells are involved in non-rheumatic sacroiliitis. However, their MR signal appearance, in any case, is the same. Therefore, our opinion is that all oedematous changes of the sacroiliac joints could be called "sacroiliitis" and, according to their aetiopathogenesis, they should be classified into rheumatic and non-rheumatic, such as mechanical or infectious.

The authors declare no conflict of interest.

\section{References}

1. Sudol-Szopinskal, KwiatkowskaB, Włodkowska-KorytkowskaM, et al. Diagnostics of sacroiliitis according to ASAS criteria: A comparative evaluation of conventional radiographs and MRI in patients with a clinical suspicion of spondyloarthropathy. Preliminary results. Pol J Radiol 2015; 80: 266-276.

2. Sieper J, Rudwaleit M, Baraliakos X, et al. The Assessment of Spondyloarthritis International Society (ASAS) handbook: a guide to assess spondyloarthritis. Ann Rheum Dis 2009; 68 (Suppl. 2): ii1-44.
3. D’Aprile P, Tarantino A. MRI of Rheumatic Spine. A Case-based Atlas. Springer 2014.

4. Banegas Illescas ME, López Menéndez C, Rozas Rodríguez ML, et al. New ASAS criteria for the diagnosis of spondyloarthritis: diagnosing sacroiliitis by magnetic resonance imaging. Radiologia 2014; 56: 7-15.

5. Berthelot J-M, le Goff B, Maugars Y, et al. Sacroiliac joint edema by MRI: Far more often mechanical than inflammatory? Joint Bone Spine 2016; 83: 3-5.

6. Vleeming A, Schuenke MD, Masi AT, et al. The sacroiliac joint: an overview of its anatomy, function and potential clinical implications. J Anat 2012; 221: 537-567.

7. Attarian DE. Septic sacroiliitis: the overlooked diagnosis. J South Orthop Assoc 2001; 10: 57-60. 